\title{
Skeletal cystic angiomatosis: A rare cause of unilateral lytic bone lesions
}

\author{
Lia Marques $^{1^{\star}}$, Elisa Vedes ${ }^{2}$, Miguel Toscano Rico $^{3}$ \\ ${ }^{1}$ Serviço de Medicina III, Hospital Pulido Valente, Centro Hospitalar Lisboa Norte, Lisboa, Portugal; \\ *Corresponding Author: marques.lia@gmail.com \\ ${ }^{2}$ Unidade de Cuidados Intensivos, Hospital da Luz, Lisboa, Portugal \\ ${ }^{3}$ Serviço de Medicina, Hospital de Santa Marta, Centro Hospitalar Lisboa Central, Lisboa, Portugal
}

Received 30 July 2013; revised 29 August 2013; accepted 10 September 2013

Copyright (C) 2013 Lia Marques et al. This is an open access article distributed under the Creative Commons Attribution License, which permits unrestricted use, distribution, and reproduction in any medium, provided the original work is properly cited.

\begin{abstract}
Cystic angiomatosis is a rare, benign, multifocal disorder of bone and viscera. Angiomatous deposits result in bone lysis and organ dysfunction. Bony cystic lesions occur in the axial and proximal appendicular skeleton. Lesions may cause bone pain or pathological fracture. Diagnosis is difficult, of exclusion and demands a biopsy. The prognosis varies upon whether the lesions are solely skeletal or there is visceral involvement. A 71-year-old man reports increasing symptoms of painful swelling in the right thoracic wall for over a month. The swelling was bony hard in consistency. Except for his bony swelling, the patient's physical examination was within normal limits, as were all his laboratory studies. X-ray imagery showed multicystic expansive lytic areas involving the right ribs. Computerized tomography, magnetic resonance imagery and gallium bone scan revealed lytic lesions of multiple right ribs, and cervical, dorsal, lumbar and sacrum iliac spine. A right rib biopsy has shown a cystic formation with endothelial walls. Five years later, the patient remained stable, with no clinical, laboratory or imagilogic progression of disease and without visceral involvement. This case is presented in his rarity and differential diagnosis challenge.
\end{abstract}

Keywords: Cystic Angiomatosis; Bone Lytic Lesions; Histiocitosis $\mathrm{X}$

\section{INTRODUCTION}

Cystic angiomatosis (CA) of bone is a rare pathological entity characterized by multifocal hemangiomatous and/or lymphangiomatous lesions of the skeleton with possible visceral organ involvement, especially the spleen [1-6]. It was first described in 1953 by Jullian E. Jacobs e Paul Kimmelstiel [6,7].

The aetiology and exact pathogenic mechanism of the disease are still unknown, but most authors believe that it is congenital malformation: a vascular hamartoma [1-3, 8,9]. It is characterized by substitution of bone tissue with hamartomatous malformation of primitive vessels disseminated and multifocal $[1,10]$.

CA affects mainly young adults of the male gender with half the cases being diagnosed around puberty [3]. Clinical signs are usually mild compared to radiological changes, which justify the fact that most of the cases of CA are revealed incidentally during radiological examination $[1,3,11]$. It is important to emphasise that a wide range of affections exists, varying from a relatively mild form in which the changes are limited to the skeleton, with the possibility of a spontaneous regression, to a very severe form with extensive involvement of extraskeletal tissues, leading at times to early death [9].

There has been a long standing confusion over the terminology of this entity: a subgroup of patients presenting with this condition present solely with skeletal lesions. This subgroup on the follow up revealed spontaneous regression of skeletal lesions [1,3,6,12]. Hence, the term "skeletal-extraskeletal angiomatosis" was coined for cases presenting with visceral manifestations [12]. Skeletal-extraskeletal angiomatosis is defined as a benign vascular proliferation involving the medullary cavity of bone and at least one other kind of tissue. It is also known as CA [12].

CA radiological changes appear most frequently in the axial skeleton in frequency order: ribs, pelvis, proximal femora, proximal humerus, spine, skull, scapulas and clavicles $[3,13]$. Hands and feet are rarely affected $[3,13]$. Radiologically the lesions appear as multiple osseous 
areas in the bone with well-defined edges surrounded by a sclerotic ring with a honey-comb appearance [3]. The intramedullary cysts are round or oval, and the cortex may appear expanded without breaking unless a pathological fracture occurs. Usually there is no periostitis [3].

Although the clinical and radiological features are generally characteristic, the process is often mistaken with other cystic lesions of the skeleton, especially histiocytosis X (Table 1). Only a wide open biopsy, preferably the partial excision of an affected rib establishes the diagnosis $[6,9]$.

Histologically angiomatosis consists of multiple dilated vascular canals, of cystic appearance and with walls lined with a layer of flat endothelial cells. The cysts, in various locations, may contain blood or proteinaceous fluid, which often makes it impossible to determine if the dilated canals are related to a haemangioma or a lymphangioma. Patients frequently have a lymphangiomatous lesion at one site and a typical haemangioma at another [6].

In the soft tissues, the angiomas are usually located in the neck or axillary regions [6], with some rare reports of intrathoracic lung masses and gastro-intestinal angiodysplasia [8,9]. Visceral involvement reaches $60 \%$ - 70\% of all cases, more frequently in the lung, spleen, liver or mediastinum [6].

Differential diagnosis of CA is presented in Table 1. A definitive diagnosis of CA is difficult to establish and is frequently one of exclusion [6]. It should be considered in patients presenting with diffuse cystic lesions of the skeleton on x-ray examination and with minimal or no associated clinical or laboratory findings [6]. A bone biopsy is needed, but is often unrevealing [3,6]. Typical hystoimmunopathologic findings show the walls of the cysts react to immunological markers of the histologic endothelium (antigens related to factor VIII and CD 31). Imagiologic methods are of limited value. Bone scan underestimates the lesions extension. Even magnetic resonance imagery (MRI) is not specific for CA. Indeed

Table 1. Differential diagnosis of cystic angiomatosis presenting as multiple lytic bone lesions.

\begin{tabular}{cc}
\hline $\begin{array}{c}\text { Differential diagnosis of cystic angiomatosis presenting } \\
\text { as multiple lytic bone lesions }\end{array}$ \\
\hline $\begin{array}{cc}\text { Common causes } \\
\text { Multiple myeloma } \\
\text { Bone Metastasis }\end{array}$ & Hystiocytosis X \\
Lymphoma & Fibrous dysplasia \\
$\begin{array}{c}\text { Hyperthyroidism } \\
\text { brown tumours }\end{array}$ & Gaucher's disease \\
Amyloidosis & Enchondromatosis \\
\hline
\end{tabular}

angiomas are variable in appearance on MRI: on T1 weighted images, the signal intensity varies from low to high, depending on the amount of adipose tissue present and the predominant tissue components-haemangioma or lymphangioma. T2 weighted images usually show areas of very high intensity corresponding to the vascular or fluid components [3].

There is no specific treatment for CA. The treatment is symptomatic. When there is osseous pain, analgesics should be prescribed and if there is increased osseous remodelling, bisphosphonates are recommended. Surgery may be an option for bone lesions. Splenectomy is to be considered if the spleen alone among the viscera is affected [1,3,6,12].

CA has a variable prognosis. Some cases, with visceral involvement, produce death in childhood [3,14,15]. When the condition is confined to the skeleton, the prognosis is good, as the lesions tend to remain static or regress $[1,3,6]$. Usually the extend of soft tissue and visceral involvement dictates the morbidity and mortality [3].

Up to 2008, no more than 200 cases of CA have been described in the literature [1]. The authors present this case report because of its extreme rarity and scientific interest concerning differential diagnosis.

\section{CASE REPORT}

A 71-year-old man was admitted to an Internal Medicine ward increasing symptoms of painful swelling in the ight posterior thoracic wall for over a month. The swelling was bony hard in consistency and painful on deep palpation. The pain was constant and worsed by any movements, but didn't wake up the patient during the night. There was no paresthesia or muscular strength alterations described. There was no anorexia or weight lost, no fever, no urinary, respiratory or gastrointestinal symptoms associated. There was no diaphoresis, no dysphonia, no dyspnea, no hemoptysis or other haemorrhage. The patient had noticed the swelling for the first time about 40 years before, but was never investigated and no specific diagnosis was made.

Previews medical story revealed multiple traumas to right ribs, smoke related chronic obstructive pulmonary disease, arterial hypertension and diabetes mellitus. His usual medications were an inalated beta 2 agonist, amlodipine and metformine. The patient had stopped smoking nine years before the admission and had a daily alcohol consumption of 20 gr ethanol.

Physical examination revealed a good performance status, apparent age was inferior to real age, no skin lesions, no lymphadenophaty, digital hipocratism or thyroid gland swelling were noticed. The right thoracic posterior wall was swollen, weak, with motion limitation and tender. There were no other inflammatory signs or 
mammary alterations. With no other abnormalities on physical examination, namely the prostatic exam was innocent.

The investigations yielded the following results: white blood cell count in the normal range, normocytic normochromic anaemia (Haemoglobin $11.5 \mathrm{~g} / \mathrm{dL}$ ), erythrocyte sedimentation rate (ESR) of $44 \mathrm{~mm} /$ first hour, lactate dehydrogenase $(\mathrm{LDH})$, alkaline phosphatase and ionised calcium in the normal range. There were no renal, hepatic or urinary abnormalities. X-ray imaging showed multicystic expansive lytic areas involving the right ribs (Figure 1).

Aetiological investigation yielded: seric proteinogram with no abnormalities, no Bence Jones protein, parathyroid and thyroid hormones in the normal range. Skeletal and skull films have shown no abnormalities. Thyroid, testicular and prostatic ultrasounds were normal. Computerized tomography (CT) has shown important lytic lesions of multiple right ribs, mainly the second rib, expansive in nature, with cortical rupture and without lesions in the surrounding soft tissues. Lytic lesion of the 6th right rib with intra thoracic growth but without pleural and pulmonary involvement. The expanding mass was multiloculated with loss of cortical definition in almost the entire length of bone with the lesion extending into the adjacent soft tissues. There was no evidence of calcification or host reaction (Figure 2). Spinal CT has shown multiple lytic bone lesions lateralized to the right (Figure 2). MRI of the spine, ribs and thorax: multiple vertebral lesions with heterogeneous sign (central low sign and peripheral high intensity sign on $\mathrm{T} 1$ and $\mathrm{T} 2$ weighted images). These lesions are of lytic nature and are more prominent in D1 to D3. Multiple structural and sign alterations over several right ribs with high intensity in T2 weighted images, compatible with CA.

A gallium bone scan has shown osteolytic and osteoblastic lesions over multiple right ribs and dorsal lumbar and sacrum iliac spine (Figure 3). Accentuated bone fixation over right ribs (1st-6th), some of these were pure lytic lesions. Other areas of anomalous fixation over 5th and 6th right condrocostal articulations, 12th dorsal vertebra, 1st and 2nd lumbar vertebrae and right sacroiliac articulation (Figure 3 ).

CT guided right rib biopsy: bloody liquid with low cellularity sediment with some histiocytes, lymphocytes and neutrophils, cystic formation with endothelial walls and without neoplastic cells. There was one giant multinucleated cell (Figure 4). A diagnosis of CA was established.

The patient was reassured, discharged, treated with nonsteroidal anti-inflammatory drugs (NSAIDs) for pain control and kept under surveillance. Five years later the patient remained stable. Laboratory evaluation has shown modest alkaline phosphatase elevation with no
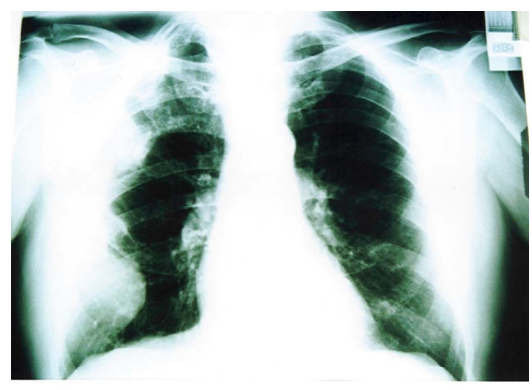

Figure 1. First thoracic radiogram.

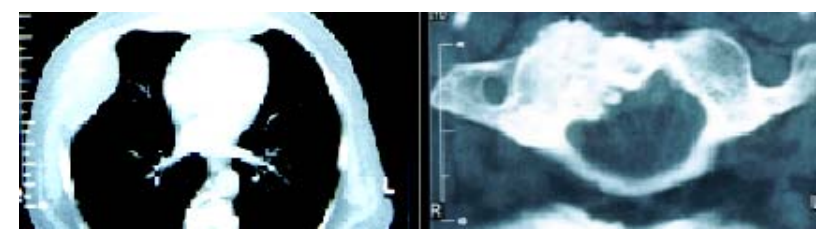

Figure 2. CT images document osteolytic lesions in right rib and second cervical vertebra.

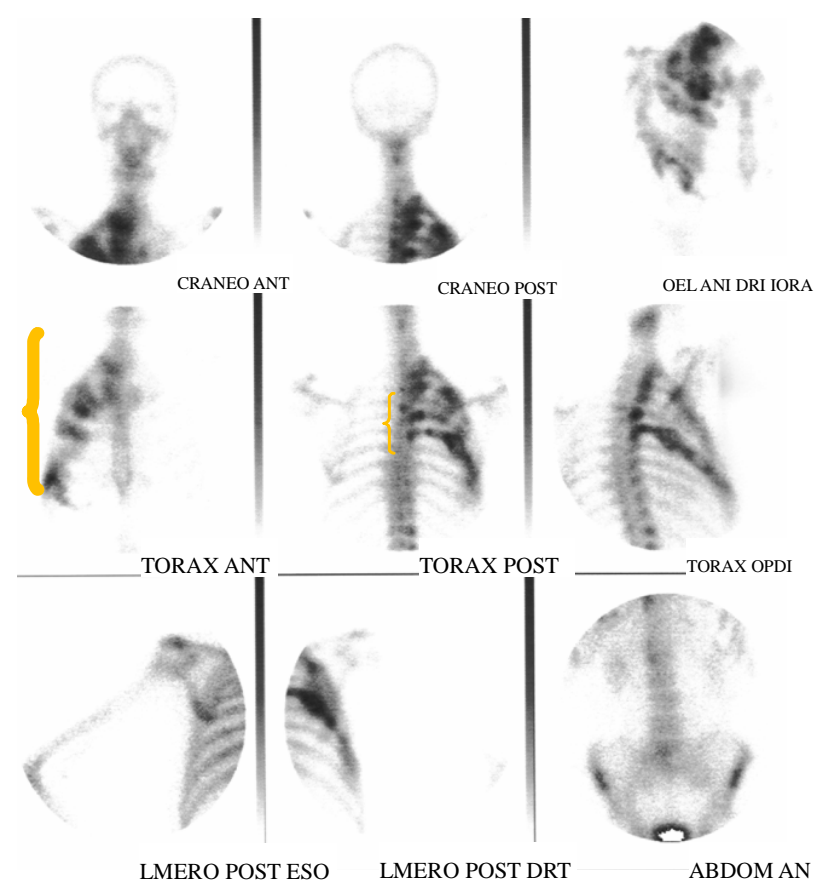

Figure 3. Bone scan with multiple right osteolytic lesions.

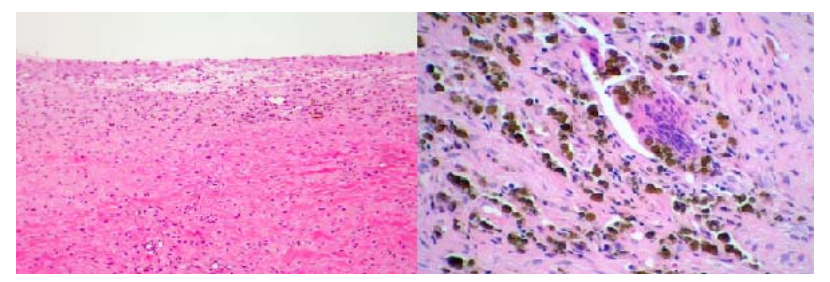

Figure 4. Right rib histopatologic characteristics - cystic formation with endothelial walls and one giant multi-nucleated cell.

other abnormalities. CT and bone scan findings has shown no progression or periosteal reaction (Figure 5). 


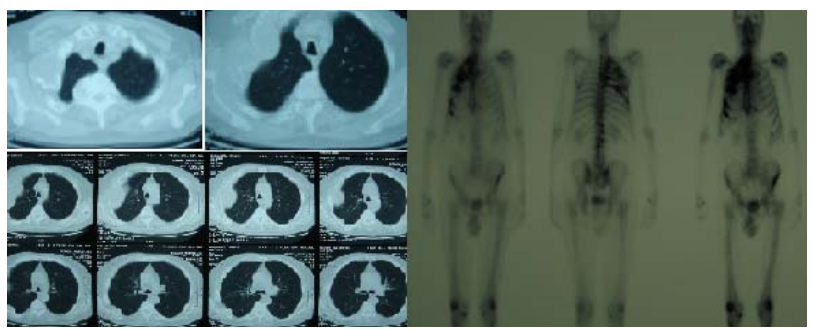

Figure 5. CT and bone scan five years after the diagnosis was established.

The patient stared therapeutic bisphosphonates with pain control and alkaline phosphatase normalization.

The patient remains stable, asymptomatic except for occasional anterior chest pain without systemic disease or visceral involvement by angiomatous lesions.

\section{DISCUSSION}

AC of the bone is a rare entity and its isolated skeletal involvement is even rarer $[6,16]$. The osseous lesions produce symptoms when a pathological fracture occurs, but as in the patient reported here, bone pain may exist without fracture $[1,3]$. The interesting feature of this case is the presence of multiple, well defined osseous lytic lesions involving the axial skeleton, that were unilateral. This bizarre appearance made the diagnosis a real challenge.

The differential diagnosis initially considered for this patient was that of multiple lytic lesion of the skeleton. The authors reinforce that one of the most important first steps in deriving a differential diagnosis when evaluating osteolytic lesions is to keep in mind the patient's age [6]. Considering the presented 71-year-old patient, the most frequent causes to be considered were metastasis and multiple myeloma (MM). These two diseases produce involvement of the flat bones such as the pelvis with a well-defined profile, but without reactional sclerosis or radiodense matrix inside. In MM, the lesions are usually the same size, that was not the case of the presented patient, and there were no laboratory abnormalities supportive of this diagnosis.

Actually, considering medical story and X-ray findings alone, in this case the diagnosis hypothesis was divided into two main groups: neoplastic and non neoplastic lesions. The first group had in favour, apart from the patient's age, raised ESR and anaemia. Favouring a benign aetiology for these osseous lesions, there were the longtime course of the swelling and the patient excellent physical status. The differential diagnoses considered in the benign group were primary and secondary hyperparathyroidism, polvostotic fibrous dysplasia, Gorham disease, histiocytosis $\mathrm{X}$ and mastocytosis. However, the clinical course, the physical examination, the laboratory findings and the pathological specimen, all helped to ex- clude these possible diagnoses. Of these, the most challenging one is histiocytosis $\mathrm{X}$ (multiple or disseminated histiocytic granuloma) which produces cysts surrounded by sclerosis in the pelvis, skull, vertebrae, and long bones, having a similar osseous distribution as CA, but with the skull bones being more often affected. The main pathological difference between CA and histocitosis $\mathrm{X}$ is that in the last one there is usually a periosteal reaction, typically absent in CA. The diagnosis must always be confirmed by histological examination of the affected bone, a rib or fibula being the most convenient for this purpose [3]. In Gorham disease (disappearing bone disease), the histology of bone lesions is very similar to those of CA, but it usually affects only one bone and produces massive bone destruction, which was not the case of this patient.

This case is similar to some of the cases of CA reported in the literature except for the late age of onset $[6,7,9,16]$. The authors highlight that the rib swelling was noticed 40 years before, at the age of 31, what is more concordant with the classic age of CA. But previous evaluation for this osseous swelling was not performed and the patient used to relate it to previous trauma.

The roentgen features in this case were also concordant with the literature, being usually lytic lesions, in sclerotic areas, expanding cysts, and generalized skeletal lucency $[9,16]$. The pathologic findings are also in agreement with the literature for CA and usually are described as a conglomeration of vascular channels in some areas, and other areas showed empty cysts with fragments of endothelial lining $[9,16]$.

There is no specific treatment, though osseous lesions can regress spontaneously. It is important to be familiarized with the clinical, radiological and pathological features of CA in order to avoid any unnecessary treatment [3].

Concerning prognosis for the presented patient, there is no compromised prognosis in the face of the patient's clinical stability, without lesions progression or visceral involvement after a five-year-surveillance-period. In this case, spontaneous regression of the cystic bone lesion may occur, as happened in some reported cases [1,3,12].

In conclusion, the authors remember this rare clinical entity and, that despite the rarity of the disease, CA should be considered as a differential diagnosis in a patient presenting with skeletal diffuse cystic lesions with minimal or no associated with clinical or laboratory findings.

\section{REFERENCES}

[1] Malik, R., Malik, R., Tandon, S. and Tanton, P. (2008) Skeletal angiomatosis-Rare cause of bone destruction: A case report with review of literature. Indian Journal of Pathology and Microbiology, 51, 515-518. 
http://dx.doi.org/10.4103/0377-4929.43745

[2] Shivaram, G.M., Pai, R.K., Ireland, K.B. and Stevens, K.J. (2007) Temporal progression of skeletal cystic angiomatosis. Skeletal Radiology, 36, 1199-1204. http://dx.doi.org/10.1007/s00256-007-0378-3

[3] Ballina-Garcia, F.J., Queiro-Silva, M.R., Molina-Suaréz, R., Fernández-Martínez, J., Rivela-Vázquez, M. and Rodríguez-Pérez, A. (1996) Multiple painful bone cysts in a young man. Annals of the Rheumatic Diseases, 55, 346349. http://dx.doi.org/10.1136/ard.55.6.346

[4] Singh, R., Grewal, D.S., Bannerjee, A.K. and Bansal, V.P. (1974) Hemangiomatosis of the skeleton. Report of a case. Journal of Bone and Joint Surgery-British Volume, 56, 136-138.

[5] Ferreti, A., Sadun, R. and Gherardi, G. (1981) Diffuse skeletal angiomatosis. Journal of Orthopaedics and Traumatology, 7, 353-358.

[6] Brower, A.C., Culver, J.E. and Keats, T.E. (1973) Diffuse cystic angiomatosis of bone. Report of two cases. American Journal of Roentgenology, 118, 456-463. http://dx.doi.org/10.2214/ajr.118.2.456

[7] Jacobs, J.F. and Kimmelstiel, P. (1953) Cystic angiomatosis skeletal system. The Journal of Bone \& Joint Surgery, 35-A, 407-420.

[8] Pulido-Zamudio, T., Orr, W. and Lertzman, M. (2001) Cystic angiomatosis of the bone appearing as intrathoracic lung masses. Canadian Respiratory Journal, 8, 187-190.

[9] Fritz Schajowicz, C.L., Aiello, M.V., Francone, R.E. and Giannini (1978) Cystic angiomatosis (hamartous haemalymphangiomatosis) of bone a clinicopathological study of three cases. The Journal of Bonne and Joint Surgery, 60-B, 100-106.

[10] Cobby, M. and Watt, I. (2003) Tumors and tumor like conditins of bone. David Sutton Text Book of Radiology and Imaging. 7th Edition, Churchill Living Stone, Philadelphia, 1287-320.

[11] Vinee, P., Tanyu, M.O., Haueastein, K.H., Sigmund, G., Stover, B. and Adler, C.P. (1994) CT and MRI of Gorham syndrome. Journal of Computer Assisted Tomography, 18, 985-989.

http://dx.doi.org/10.1097/00004728-199411000-00028

[12] Clayer, M. (2002) Skeletal angiomatosis in association with gastro-intestinal angiodysplasia and paraproteinemia: A case report. The Journal of Orthopaedic Surgery (Hong Kong), 10, 85-88.

[13] Conway, W.F. and Hayes, C.W. (1993) Miscellaneous lesions of bone. Radiologic Clinics of North America, 34, 324-328.

[14] Gilsanz, V., Yeh, H.-C. and Baron, M.G. (1976) Multiple lymphangiomas of the neck, axilla, mediastinum and bones in an adult. Radiology, 120, 161-162.

[15] Watts, M.A., Gibbons, J.A. and Aaron, B.L. (1982) Mediastinal and osseus lymphangiomatosis: Case report and review. The Annals of Thoracic Surgery, 34, 324-328. http://dx.doi.org/10.1016/S0003-4975(10)62504-0

[16] Wallis, L.A., Asch, T. and Maisel, B.W. (1964) Diffuse skeletal hemangiomatosis: Report of two cases and review of literature. American Journal of Medicine, 37, 545-563. http://dx.doi.org/10.1016/0002-9343(64)90068-3 